\title{
Vacunación contra el virus de la hepatitis B en recién nacidos de mujeres peruanas participantes de la Encuesta Demográfica y de Salud Familiar, 2016
}

Vaccination against the hepatitis B virus in newborns of peruvian women participating in the demographic and family health survey, 2016

\author{
Joel Christian Roque Henriquez ${ }^{1,2}$, Jhotsen David Mera Villarreal ${ }^{1}$, Franco Ronald Romani Romani ${ }^{2}$ \\ ${ }^{1}$ Universidad de San Martín de Porres. Lima, Perú. \\ ${ }^{2}$ Oficina General de Investigación y Transferencia Tecnológica, Instituto Nacional de Salud. Lima, Perú.
}

An Fac med. 2018;79(3):218-24. / http://dx.doi.org/10.15381/anales.v79i3.15314

Correspondencia:

Franco Ronald Romani Romani

fromani@ins.gob.pe

Recibido: 19 de setiembre 2018

Aprobado: 29 de setiembre 2018

Conflictos de interés: Los autores

declaran no tener conflictos de interés

Fuentes de financiamiento:

Instituto Nacional de Salud

Artículo realizado en base a la tesis presentada por Jhotsen David Mera Villarreal para optar el título de médico cirujano en la Universidad de San Martín de Porres

Citar como: Roque J, Mera J, Romani $F$. Vacunación contra el virus de la hepatitis $B$ en recién nacidos de mujeres peruanas participantes de la Encuesta Demográfica y de Salud Familiar, 2016. An Fac med. 2018;79(3):218-24.

DOI: http://dx.doi.org/10.15381/anales. v79i3.15314

\section{Resumen}

Introducción. El esquema nacional de vacunación del Perú incluye la vacuna contra hepatitis $B$ en recién nacidos; la Organización Mundial de Salud ha establecido metas para la cobertura de dicha vacunación. El objetivo del estudio fue estimar la proporción de recién nacidos de mujeres peruanas participantes de la Encuesta Demográfica y de Salud Familiar (ENDES) del 2016, que recibieron la primera dosis de la vacuna contra hepatitis B al nacer. Métodos. Se realizó un estudio de fuente secundaria, la unidad informante fueron las mujeres en edad fértil de 15 a 49 años, que reportaron tener niños menores de cinco años, cuyo peso al nacer haya sido de $2000 \mathrm{~g}$ o más, y que contaban con datos referentes a vacunación. Los datos sobre el estado de vacunación contra hepatitis B se obtuvieron a partir de la tarjeta de vacunación del niño menor de cinco años. Los resultados son presentados para el nivel nacional, regional, y según algunos subgrupos especificos. Resultados. A nivel nacional, el 67,5\% (IC95\%: 66,3-68,6) de recién nacidos de mujeres participantes de la ENDES 2016 recibieron la vacunación contra hepatitis B. La región con mayor cobertura fue Huánuco con 84,3\% (IC95\%: 80,8-87,2). Entre los recién nacidos en establecimientos de salud públicos, la vacunación llegó al $72,6 \%$, mientras que en establecimientos de salud privados fue el $43,2 \%$. Conclusiones. La proporción de recién nacidos que recibieron la vacunación contra hepatitis $B$ es variable según región, siendo esta mayor entre aquellos que nacieron en establecimientos de salud públicos.

Palabras clave: Hepatitis B; Recién nacido; Vacunación; Cobertura de vacunación; Perú

\section{Abstract}

Introduction. The national vaccination scheme in Peru includes the hepatitis B vaccine at birth, the World Health Organization established goals for the coverage of this vaccination. The objective of the study was to estimate the proportion of newborns of women participating in the Demographic and Family Health Survey (ENDES) of Peru in 2016, who received vaccination against hepatitis B. Methods. A secondary study was conducted, the informants subject were childbearing age women from 15 to 49 years old, who reported having a child under five years of age, whose weight at birth was $2000 \mathrm{~g}$ or more, and who have data regarding vaccination. The data on vaccination status against hepatitis $B$ was obtained from the vaccination card. The results are presented for the national, regional level, and according to some specific sub groups. Results. At the national level, $67,5 \%$ (95\% Cl: 66,3-68,6) of newborns received vaccination against hepatitis B. The region with the highest coverage was Huanuco with $84,3 \%$ (95\% Cl: 80,8-87,2). Among newborns in public health facilities, vaccination reached $72,6 \%$, while in private health facilities was $43,2 \%$. Conclusions. The proportion of newborns who received vaccination against hepatitis $B$ varies according to region, being higher among those who were born in public health facilities.

Keywords: Hepatitis B; Newborn; Vaccination; Vaccination coverage; Peru 


\section{INTRODUCCIÓN}

La Agenda para el Desarrollo Sostenible establece que al 2030 se debe poner fin a epidemias como el SIDA, tuberculosis, malaria, además de combatir la hepatitis ${ }^{1}$. La Organización Mundial de la Salud (OMS) formuló una estrategia mundial cuya meta es reducir los casos de infección por hepatitis $B$ y $C$ a menos de un millón para el 2030². En el 2014, la Asamblea Mundial de Salud estableció la necesidad de reducir la prevalencia de la infección crónica por hepatitis $B(H \vee B)$, a través de la reducción de la transmisión perinatal mediante la vacunación en recién nacidos ${ }^{3}$. Matemáticamente se ha estimado que sin dicha vacunación se producirían al 2020, 25 millones de casos nuevos, lo cual implica que dicha intervención ya ha reducido un 83\% el número de casos nuevos de infección por HvB ${ }^{4}$. En el Perú, se ha observado en una región endémica de $\mathrm{HvB}$, que un programa piloto de vacunación implementado en 1991 logró reducir la tasa de mortalidad por hepatocarcinoma, cirrosis y hepatitis fulminante; dicho efecto fue particularmente importante en menores de 15 años 5

El esquema nacional de vacunación del Perú incluye la vacuna contra HvB; la primera dosis debe ser aplicada a recién nacidos a término de $2000 \mathrm{~g}$ o más, antes de las doce y máximo dentro de las primeras 24 horas de vida, con la finalidad de prevenir la infección vertical de $\mathrm{HvB}^{6}$. Su efectividad disminuye progresivamente si se administra días posteriores al nacimiento, siendo aún efectiva hasta siete días luego del nacimiento ${ }^{7}$. En el Perú, la cobertura de vacunación ha sido determinada por información de tipo administrativo remitida por los establecimientos de salud. Dichas coberturas variaron de $74 \%(2010)$ a $82 \%(2013)^{8}$, superiores a la meta establecida por la OMS para el $2020(50 \%)^{9}$. A nivel de hospitales y clínicas de Lima Metropolitana y Callao, usando datos obtenidos de historias clínicas materno y neonatal se encontró que la cobertura de vacunación antes de las 24 horas fue cercana al $90 \%{ }^{10}$.

El monitoreo de la cobertura de vacunación usando datos y reportes administrativos de los establecimientos de salud tienen desventajas como: no contar con la adecuada cantidad de población expuesta (denominador), no reporta información del sector privado, es susceptible de sobreestimación por estar vinculada a pagos por incentivos, puede tener errores de transcripción especialmente si no se usa registro electrónico. Otra alternativa son las estimaciones obtenidas a partir de encuestas poblacionales como la Encuesta Demográfica y de Salud Familiar (ENDES), la cual utiliza técnica de muestro probabilística que permite estimar coberturas para sub-grupos específicos y permiten medir otros indicadores demográficos, o de acceso a servicios de salud $^{11}$. En conclusión, las coberturas estimadas a partir de datos administrativos podrían estar sobreestimadas, mientras que las estimaciones obtenidas a partir de encuestas poblacionales son más válidas y confiables ${ }^{12}$.

El presente estudio estimó la proporción de recién nacidos de madres participantes en la ENDES 2016, que recibieron la vacuna contra la HvB al nacer; también exploró algunos factores asociados a dicha vacunación. Esta información permitirá evaluar el avance del Perú en la prevención de la transmisión madre-niño de HvB, considerando que en el 2015 a nivel mundial la cobertura de vacunación al nacimiento alcanzó 39\% y en las Américas dicha cobertura fue de $72 \%$.

\section{MÉTODOS}

Se realizó un estudio de fuente secundaria. La fuente de información fue la ENDES del año 2016, ejecutada por el Instituto Nacional de Estadística e Informática (INEI). La ENDES tiene un muestreo complejo que se caracteriza por ser bietápico, probabilístico de tipo equilibrado, estratificada e independiente, a nivel departamental y por área urbana y rural. El diseño muestral permite inferencias a nivel nacional y regional, así como en el ámbito rural y urbano, y para las tres regiones naturales (costa, sierra y selva). El tamaño muestral de la ENDES fue de 35910 viviendas, de las cuales 14160 estuvieron ubicadas en capitales de departamento y los 43 distritos que conforman la provincia de Lima, $9300 \mathrm{del}$ resto urbano y 12450 del área rural. La base de datos se encuentra disponible en forma gratuita en la página web del INEI (http://iinei.inei.gob.pe/microdatos/).

Para el presente estudio usamos el cuestionario individual de la ENDES, el cual tiene como unidad informante a mujeres en edad fértil entre 15 y 49 años de edad. Dicho cuestionario incluye preguntas sobre: características demográficas y sociales, historia reproductiva, conocimiento y uso de anticonceptivos, atención pre-natal, asistencia del parto, embarazo y lactancia, inmunización entre otros. Los criterios de inclusión de las unidades de análisis fueron: niño menor de cinco años, con 2000g o más de peso al nacer (variable M19- módulo REC41) y contar con datos referentes a vacunación contra hepatitis $B$ al nacimiento (variable S45B0 - módulo REC95). En caso de mujeres con más de un hijo menor de cinco años, se analizó los datos por cada uno de ellos. La vacunación al nacer contra la HvB fue introducida en el Perú en 2003, por lo cual la muestra estudiada estuvo expuesta a la intervención entre 2011 y 2015.

La información sobre inmunización contra HvB fue obtenida a partir de la tarjeta de vacunación del recién nacido (con o sin fecha consignada). Se optó por dicha definición debido a que ha mostrado mejor rendimiento para medir cobertura de vacunación (concordancia de $81 \%$, sensibilidad $77 \%$, especificidad $84 \%$ y valor predictivo positivo de $92 \%)^{13}$. Para fines comparativos, también presentamos la estimación de la cobertura de vacunación según información obtenida de la tarjeta de vacunación y del reporte de la madre ${ }^{14}$.

Las variables independientes fueron: nivel educativo de la madre (postgrado, superior universitaria, superior no universitaria, secundaria, primaria, ninguno/pre-escolar), estado civil actual de la madre (casada, conviviente, viuda, divorciada, separada, nunca unida), aseguramiento en salud (sí/no), región natural (resto de costa, Lima Metropolitana, sierra, selva), tipo de lugar de residencia (urbano/rural), lugar de residencia de facto (capital, gran ciudad; pequeña cuidad; pueblo; campo), índice de riqueza (más rico, rico, medio, pobre, más pobre), región política (las 25 regiones del país), número de controles prenatales adecuados (seis o más: sí; 
menos de 6: no), lugar de nacimiento del niño (domicilio, establecimiento público, privado, organismo no gubernamental, otros), y trabajo en los últimos doce meses (sí/no).

Se estimaron las frecuencias relativas de las características de la muestra con sus respectivos intervalos de confianza al 95\%. De igual forma se estimó el cumplimiento de la vacunación contra la HvB en recién nacidos a nivel total y por regiones, así como para las categorías de las variables analizadas. La comparación de proporciones para muestras independientes se realizó mediante chi cuadrado. Un valor de $p$ menor de 0,05 fue considerado como estadísticamente significativo. Se realizó el análisis estadístico para muestras complejas, los instrumentos de ajustes utilizados fueron factor de ponderación (factor mujer: v005), número de estrato de la muestra (v022) y número de conglomerado (v001), ubicados en el módulo REC0111. Se utilizó el paquete estadístico Stata 14.0.

La base de datos de la ENDES 2016 es de acceso público, y respeta la confidencialidad de datos de los participantes. El presente trabajo fue aprobado por el Comité Institucional de Ética en Investigación de la Universidad San Martín de Porres.

\section{RESULTADOS}

De 21175 niños menores de cinco años, 233 fueron excluidos debido a que no contaban con datos para la variable de vacunación contra la HvB al nacimiento, 292 tuvieron peso al nacer menor de 2000 y y 936 no tenían información sobre el peso al nacer. Cumplieron los criterios de inclusión 19714.

La media de edad de las madres de los niños menores de cinco años fue de 30,2 años (IC95\%: 30,0-30,3), el 47,3\% culminó los estudios secundarios, 84,7\% reportó contar con seguro de salud. El $10,8 \%$ refirió haber tenido seis o más controles prenatales, $81,2 \%$ refirió haber tenido el parto en establecimientos de salud públicos (tabla 1). Se encontró asociación estadísticamente significativa entre lugar de nacimiento del niño con índice de riqueza $(p<0,001)$, lugar de residencia (incluyendo la de facto) $(p<0,001)$ y contar con seguro de salud $(p<0,001)$.
Tabla 1. Características sociodemográficas de las madres informantes y participantes en la Encuesta Demográfica y de Salud Familiar (ENDES), 2016.

Característica Estimación puntual (\%) IC $95 \%$

Edad de la madre (años)

15-19

20-24

4,7

19,6

25-29

24,2

$30-34$

23,6

35-39

17,2

40-44

45-49

8,6

2,0

Educación de la madre

Post-grado

Superior universitaria

1,3

Superior no universitaria

12,9

17,3

Secundaria

Primaria

Ninguno/pre-escolar

47,3

19,8

1,3

Estado civil actual de la madre

Casada

Conviviente

26,4

58,9

Viuda

0,2

Divorciada

Separada

Nunca unida

Cuenta con seguro de salud Sí

Seguro privado

EsSalud/IPSS

Fuerzas armadas o policiales

Seguro Integral de Salud (SIS)

Entidad prestadora de salud

No

Región natural

Lima Metropolitana

Resto de costa

Selva

Sierra

Tipo de lugar de residencia

Urbano

Rural

Lugar de residencia de facto

Capital, gran ciudad

Pequeña ciudad

Pueblo

Campo

Índice de riqueza

Más rico

Rico

Medio

Pobre

Más pobre

Controles prenatales ( $\geq 6$ controles) Sí

No

Lugar de nacimiento del niño

Su domicilio

Sector público

Sector privado

Organismos no gubernamentales

Otro

0,1

4,8

84,7

1,4

24,8

0,7

58,3

2,5

15,3

29,7

26,5

16,9

26,9

75,3

24,7

29,7

21,3

24,3

24,7

16,5

18,4

21,6

23,7

19,8

10,8

89,2

5,0

81,2

12,7

0,3

0,7

67,2

32,8

4,3-5,1

18,8-20,5

23,3-25, 1

$22,7-24,6$

$16,3-18,0$

$8,1-9,2$

$1,8-2,3$

1,0-1,7

$11,9-13,9$

$16,5-18,2$

46,0-48,6

18,8-20,9

$1,1-1,6$

$25,3-27,6$

57,6-60,1

$0,1-0,3$

$0,1-0,2$

9,0-10,2

$4,4-5,2$

$83,9-85,5$

$1,1-1,8$

23,6-26,0

0,5-0,9

56,9-59,6

$1,9-3,3$

$14,5-16,1$

27,3-32,2

24,7-28,5

15,4-18,4

$25,1-28,8$

$73,9-76,6$

$23,4-26,1$

27,3-32,2

$19,8-22,8$

$23,1-25,5$

$23,4-26,1$

$15,1-18,0$

17,4-19,4

20,6-22,7

$22,5-25,0$

$18,6-21,1$

10,1-11,6

$88,4-89,9$

4,4-5,8

$79,9-82,5$

$11,6-13,9$

$0,2-0,5$

0,4-1,1

Trabajó en los últimos 12 meses

$66,1-68,3$

$31,7-33,9$ 
El 67,5\% (IC95\%: 66,3-68,6) de los niños recibió la vacunación contra la HvB al nacimiento. Dicha proporción llegó al $71,7 \%$ y $73,7 \%$ en niños de madres con educación primaria y ninguno/preescolar, respectivamente. La vacunación en niños cuyas madres contaban con Seguro Integral de Salud (SIS) fue 71,6\%, mientras que entre las que tuvieron seguro privado fue del 44,7\%. En niños de mujeres del ámbito rural se verificó que $72 \%$ recibió la vacunación, dicha proporción es congruente con lo encontrado en niños de mujeres cuya residencia de facto es el campo (72\%). En los niños nacidos en establecimientos de salud públicos, la vacunación llegó al 72,6\%, mientras que en aquellos que nacieron en establecimientos de salud privados alcanzó el 43,2\% (tabla 2).

En el análisis según regiones, tres regiones (Piura, Lambayeque y Callao) tuvieron coberturas menores al $60 \%$, y diez (Tumbes, Amazonas, Loreto, Lima, Ica, Arequipa, Madre de Dios, Moquegua, Tacna y Puno) tuvieron coberturas entre $60 \%$ y $69 \%$. La región con mayor cobertura fue Huánuco 84,3\% (IC95\%: 80,8$87,2)$. A nivel nacional, la diferencia entre las coberturas obtenidas considerando el reporte de la madre es de 18,3 puntos porcentuales (pp). Las mayores diferencias entre las estimaciones puntuales de cobertura se observan en las regiones de Ica, Tacna y Moquegua (mayor a 28 pp), mientras que en Huánuco dicha diferencia fue la menor (6,6 pp). (Tabla 3).

\section{DISCUSIÓN}

En el periodo 2011 al 2015, el 67,5\% de recién nacidos vivos recibieron la vacunación contra la HvB de acuerdo al esquema nacional de vacunación. Dicha cobertura difiere a lo encontrado por Ropero y col. ${ }^{8}$ quienes reportaron que la cobertura en Perú fue del $82 \%$ en 2013, $78 \%$ en 2014 y $79 \%$ en 2015 . Nuestra estimación también es menor al 88,9\% de cobertura encontrada en hospitales de Lima Metropolitana y Callao ${ }^{10}$. Las diferencias son explicadas por los diferentes métodos de estimación de cobertura empleados. Es conocido que las coberturas obtenidas a partir de datos administrativos rutinariamente recogidos sobreestiman los datos, comparada con aquellas obtenidas en encuestas poblacionales en hogares. Otra explicación sería el periodo analizado; así, las investigaciones al respecto se realizan anualmente. En nuestro estudio se reporta la estimación para un periodo temporal de cinco años.

En nuestro estudio, la estimación encontrada tuvo como numerador a los niños menores de cinco años en quienes se pudo constatar la primera dosis contra HvB a partir de la tarjeta de vacunación solicitada a la madre. Por otro lado, el denominador incluyó sólo a aquellos en quienes la vacuna estuvo indicada, es decir aquellos con peso al nacimiento de 2000 g o más. Dichas definiciones fueron empleadas para reducir la posibilidad de sesgos de mala clasificación.

Cabe resaltar que la confiabilidad de las estimaciones sobre cobertura de inmunización obtenidas en encuestas de hogares varía en función de si la información sobre vacunación es tomada directamente de la tarjeta de vacunación o si es reportada por la madre. En nuestro estudio dichas diferencias varían según región; sin embargo, a nivel nacional, usando el reporte de la madre en forma adicional a la tarjeta de vacunación la cobertura llegaría a 85,8\%, lo cual podría estar sobreestimando la real ejecución de la vacunación en recién nacidos. Dicho sesgo en la medición generado por el autoreporte podría ser ocasionado por un sesgo de deseabilidad o aceptación social; y para el caso de nacimientos más antiguos por un sesgo de memoria.

Respecto a las coberturas en subgrupos específicos, resalta que entre las mujeres que tuvieron SIS, el 71,5\% de sus recién nacidos recibió la vacunación. Dichas proporciones son mayores comparado con mujeres que contaban con seguro privado, seguro social o atendidas en una entidad prestadora de salud. También encontramos una mayor cobertura en niños de madres residentes en la sierra y selva; lo cual explicaría las mayores coberturas encontradas en la zona rural y en los quintiles más pobres, características comunes de la sierra y selva del Perú.

A nivel de la implementación, encontramos que la ejecución de esta inmunización sería mayor cuando el parto se realiza en establecimientos del sector público (72,6\%). Por el contrario, en situaciones en que el parto se realiza en el domicilio o en establecimientos del sector privado, la cobertura de la vacunación contra HvB sería menor al 50\%. Las diferencias de cobertura de este tipo de vacunación según esta característica ha sido reportadas por otros estudios ${ }^{14,15}$

En Colombia, un estudio realizado entre 2011 y 2013, y cuyo registro de la vacunación fue a partir de la tarjeta, se encontró que entre aquellos que nacieron en establecimientos de salud la cobertura alcanzó el 72,5\%, proporción similar a la encontrada en nuestro estudio, para el caso de establecimientos del sector público. En dicho estudio, el nacer en un establecimiento de salud fue el único factor modificable independientemente asociado a la vacunación contra HvB al nacimiento ${ }^{15}$. Otro estudio en Papua Nueva Guinea en 2014, también reporta asociación significativa entre la vacunación y parto institucional ${ }^{16}$. Una correlación positiva entre parto institucional y vacunación contra HvB al nacer fue reportada en un estudio ecológico a nivel de países ${ }^{17}$. Estos hallazgos brindan evidencia a nivel ecológico e individual que la promoción del parto institucional mejoraría la cobertura de la vacunación contra HvB al nacer.

En nuestro estudio, encontramos que la cobertura de vacunación es superior al $70 \%$ en los quintiles pobre y más pobre; dicho hallazgo podría ser explicado debido a que dicha población es beneficiaria del Seguro Integral de Salud, cobertura que les permite acceder al parto institucional en establecimientos de salud públicos. Este hallazgo es consistente con lo encontrado en un estudio que evaluó la vacunación de HvB en la Encuesta Demográfica y de Salud de Camerún. En dicho estudio, la probabilidad de vacunación se incrementa a mayor nivel de pobreza ${ }^{14}$.

Por otro lado, existen una serie de características de las mujeres que se comportarían como variables confusoras para la asociación entre parto institucional y vacunación al nacimiento. Entre dichas características estarían el ser residente de la selva o sierra, el vivir en el área rural, en el campo, y el aseguramiento en salud. La evidencia generada por un es- 
Tabla 2. Proporción de vacunación contra hepatitis B en recién nacidos de madres enroladas en la Encuesta Demográfica y de Salud Familiar (ENDES) 2016, según características sociodemográficas.

\begin{tabular}{|c|c|c|c|c|}
\hline \multirow[t]{2}{*}{ Característica } & $\begin{array}{c}\text { Registro a partir de tarjeta } \\
\text { de vacunación }\end{array}$ & Valor de $p$ & $\begin{array}{l}\text { Registro a partir de tarjeta + } \\
\text { reporte de madre* }\end{array}$ & Valor de $p$ \\
\hline & $\%$ (IC 95\%) & & $\%$ (IC 95\%) & \\
\hline Edad de la madre (años) & & $<0,01$ & & 0,02 \\
\hline $15-19$ & $72,2(68,3-75,9)$ & & $88,6(85,7-91,1)$ & \\
\hline $20-24$ & $68,7(66,6-70,7)$ & & $87,4(85,9-88,8)$ & \\
\hline $25-29$ & $68,8(66,8-70,7)$ & & $86,5(85,0-87,9)$ & \\
\hline $30-34$ & $67,1(64,8-69,4)$ & & $84,4(82,4-86,3)$ & \\
\hline $35-39$ & $63,4(60,6-66,1)$ & & $84,1(81,9-86,1)$ & \\
\hline $40-44$ & $68,4(65,2-71,4)$ & & $86,1(83,5-88,4)$ & \\
\hline $45-49$ & $63,7(57,0-69,9)$ & & $85,2(79,6-89,5)$ & \\
\hline Educación de la madre & & $<0,01$ & & $<0,01$ \\
\hline Post-grado & $58,1(45,7-69,7)$ & & $81,8(72,4-88,6)$ & \\
\hline Superior universitaria & $58,8(55,4-62,0)$ & & $81,7(78,7-84,4)$ & \\
\hline Superior no universitaria & $66,5(63,9-69,0)$ & & $84,8(82,2-87,0)$ & \\
\hline Secundaria & $68,5(67,2-69,9)$ & & $87,7(86,6-88,6)$ & \\
\hline Primaria & $71,7(69,5-73,8)$ & & $85,3(83,5-87,0)$ & \\
\hline Ninguno/pre-escolar & $73,7(66,1-80,1)$ & & $85,9(78,7-90,9)$ & \\
\hline Estado civil actual de la madre & & $<0,01$ & & 0,64 \\
\hline Casada & $67,0(64,8-69,2)$ & & $84,9(83,0-86,6)$ & \\
\hline Conviviente & $69,2(67,8-70,5)$ & & $86,4(85,3-87,4)$ & \\
\hline Viuda & $39,5(23,4-58,2)$ & & $82,8(62,1-93,4)$ & \\
\hline Divorciada & $55,3(25,5-81,8)$ & & $78,3(43,8-94,3)$ & \\
\hline Separada & $58,2(54,9-61,5)$ & & $85,3(82,4-87,8)$ & \\
\hline Nunca unida & $69,2(64,7-73,4)$ & & $85,6(81,6-88,9)$ & \\
\hline \multicolumn{5}{|l|}{ Cuenta con seguro de salud } \\
\hline Seguro privado & & $<0,01$ & & $<0,01$ \\
\hline Sí & $44,7(34,5-55,4)$ & & $68,8(57,1-78,5)$ & \\
\hline No & $67,8(66,7-68,9)$ & & $86,1(85,1-86,9)$ & \\
\hline EsSalud/IPSS & & $<0,01$ & & 0,05 \\
\hline Sí & $64,5(62,1-66,9)$ & & $84,4(82,4-86,2)$ & \\
\hline No & $68,5(67,2-69,7)$ & & $86,3(85,3-87,2)$ & \\
\hline Fuerzas armadas o policiales & & 0,99 & & 0,99 \\
\hline Sí & $67,4(56,0-77,1)$ & & $85,9(74,9-92,5)$ & \\
\hline No & $67,5(66,3-68,6)$ & & $85,8(83,0-85,0)$ & \\
\hline Seguro Integral de Salud (SIS) & & $<0,01$ & & $<0,01$ \\
\hline Sí & $71,6(70,3-72,8)$ & & $87,4(86,4-88,3)$ & \\
\hline No & $61,8(60,0-63,6)$ & & $83,7(82,2-85,1)$ & \\
\hline Entidad prestadora de salud & & $<0,01$ & & $<0,01$ \\
\hline Sí & $35,5(26,1-46,2)$ & & $60,0(49,3-69,9)$ & \\
\hline No & $68,3(67,2-69,4)$ & & $86,5(85,6-87,3)$ & \\
\hline Región natural & & $<0,01$ & & $<0,01$ \\
\hline Lima Metropolitana & $61,5(58,7-64,2)$ & & $81,4(79,0-83,6)$ & \\
\hline Resto de costa & $64,4(62,6-66,2)$ & & $84,9(83,3-86,4)$ & \\
\hline Selva & $70,6(68,3-72,8)$ & & $85,3(83,4-87,0)$ & \\
\hline Sierra & $75,2(73,6-76,8)$ & & $91,9(91,0-92,8)$ & \\
\hline Tipo de lugar de residencia & & $<0,01$ & & $<0,01$ \\
\hline Urbano & $66,0(64,7-67,3)$ & & $85,1(84,0-86,2)$ & \\
\hline Rural & $72,0(69,8-74,0)$ & & $88,0(86,3-89,4)$ & \\
\hline Lugar de residencia de facto & & $<0,01$ & & $<0,01$ \\
\hline Capital, gran ciudad & $61,5(58,7-64,2)$ & & $81,4(79,0-83,6)$ & \\
\hline Pequeña ciudad & $69,8(68,0-71,6)$ & & $88,1(86,5-89,5)$ & \\
\hline Pueblo & $68,3(66,5-70,0)$ & & $87,1(85,7-88,4)$ & \\
\hline Campo & $72,0(69,8-74,0)$ & & $88,0(86,3-89,4)$ & \\
\hline
\end{tabular}




\begin{tabular}{|c|c|c|c|c|}
\hline \multirow[t]{2}{*}{ Característica } & $\begin{array}{c}\text { Registro a partir de tarjeta } \\
\text { de vacunación }\end{array}$ & Valor de $p$ & $\begin{array}{l}\text { Registro a partir de tarjeta + } \\
\text { reporte de madre* }\end{array}$ & \multirow[t]{2}{*}{ Valor de $p$} \\
\hline & $\%$ (IC 95\%) & & $\%$ (IC 95\%) & \\
\hline Índice de riqueza & & $<0,01$ & & $<0,01$ \\
\hline Más rico & $58,6(54,9-62,2)$ & & $78,9(75,5-81,9)$ & \\
\hline Rico & $66,3(63,9-68,6)$ & & $86,6(84,7-88,3)$ & \\
\hline Medio & $67,7(65,6-69,7)$ & & $87,0(85,4-88,4)$ & \\
\hline Pobre & $71,3(69,6-73,0)$ & & $89,0(87,7-90,2)$ & \\
\hline Más pobre & $71,1(68,8-73,4)$ & & $85,8(83,8-87,5)$ & \\
\hline № de controles prenatales adecuados & & $<0,01$ & & $<0,01$ \\
\hline Sí & $58,4(55,3-61,3)$ & & $79,6(77,0-81,9)$ & \\
\hline No & $68,6(67,4-69,8)$ & & $86,6(85,6-87,5)$ & \\
\hline Lugar de nacimiento del niño & & $<0,01$ & & $<0,01$ \\
\hline Su domicilio & $50,3(44,8-55,7)$ & & $67,5(62,7-72,0)$ & \\
\hline Sector público & $72,6(71,6-73,6)$ & & $90,5(89,8-91,1)$ & \\
\hline Sector privado & $43,2(39,7-46,8)$ & & $64,2(60,6-67,7)$ & \\
\hline Organismos no gubernamentales & $63,4(44,2-79,1)$ & & $74,6(57,2-86,6)$ & \\
\hline Otro & $41,4(23,0-62,5)$ & & $75,6(57,7-87,6)$ & \\
\hline Trabajó en los últimos 12 meses & & $<0,01$ & & 0,14 \\
\hline Sí & $65,8(64,4-67,1)$ & & $86,2(85,2-87,2)$ & \\
\hline No & $71,0(69,2-72,8)$ & & $85,0(83,5-86,4)$ & \\
\hline
\end{tabular}

* Sí: Vacunación (con fecha) consignada en la tarjeta de vacunación + vacunación (sin fecha) consignada en la tarjeta de vacunación + reporte de la madre

tudio pre-pos intervención a nivel comunitario realizado en un país endémico de HvB muestra que, mejorando el acceso al parto en establecimientos de salud, se mejora la cobertura de la dosis al nacer contra la HvB. La intervención consistió
Tabla 3. Cumplimiento de la vacunación contra Hepatitis B en recién nacidos de madres participantes en la Encuesta Demográfica y de Salud Familiar (ENDES) 2016, según región política.

\begin{tabular}{|c|c|c|c|}
\hline \multirow[t]{2}{*}{ Región política } & $\begin{array}{c}\text { Registro a partir de tarjeta } \\
\text { de vacunación } \\
\text { (a) }\end{array}$ & $\begin{array}{l}\text { Registro a partir de tarjeta } \\
\text { + reporte de madre* } \\
\text { (b) }\end{array}$ & $\begin{array}{c}\text { Diferencias entre } \\
\text { estimaciones puntuales } \\
\text { b - a }\end{array}$ \\
\hline & $\%($ IC $95 \%)$ & $\%($ IC $95 \%)$ & \\
\hline Amazonas & $65,1(60,1-69,8)$ & $80,4(76,2-83,9)$ & 15,3 \\
\hline Ancash & $79,3(75,6-82,6)$ & $91,9(89,4-93,8)$ & 12,6 \\
\hline Apurímac & $82,5(79,4-85,2)$ & $98,9(97,9-99,4)$ & 16,4 \\
\hline Arequipa & $69,2(65,0-73,0)$ & $92,4(89,9-94,3)$ & 23,2 \\
\hline Ayacucho & $80,6(77,1-83,7)$ & $93,4(91,2-95,1)$ & 12,8 \\
\hline Cajamarca & $72,4(67,8-76,6)$ & $88,4(85,4-90,9)$ & 16,0 \\
\hline Callao & $55,8(51,7-59,8)$ & $78,7(75,4-81,7)$ & 22,9 \\
\hline Cusco & $81,9(78,0-85,2)$ & $95,0(92,3-96,7)$ & 13,1 \\
\hline Huancavelica & $80,7(76,7-84,2)$ & $97,2(95,6-98,2)$ & 16,5 \\
\hline Huánuco & $84,3(80,8-87,2)$ & $90,9(88,2-93,0)$ & 6,6 \\
\hline Ica & $60,4(56,2-64,4)$ & $89,1(86,7-91,2)$ & 28,7 \\
\hline Junín & $71,4(66,6-75,8)$ & $88,9(86,1-91,2)$ & 17,5 \\
\hline La Libertad & $75,5(71,6-79,1)$ & $91,3(88,8-93,3)$ & 15,8 \\
\hline Lambayeque & $57,7(53,4-61,8)$ & $80,3(76,5-83,6)$ & 22,6 \\
\hline Lima & $63,2(60,4-65,9)$ & $82,9(80,5-85,1)$ & 19,7 \\
\hline Loreto & $65,7(60,0-71,1)$ & $79,6(74,4-84,0)$ & 13,9 \\
\hline Madre de Dios & $68,5(64,6-72,1)$ & $94,8(92,8-96,3)$ & 26,3 \\
\hline Moquegua & $65,4(61,4-69,2)$ & $93,5(91,0-95,3)$ & 28,1 \\
\hline Pasco & $73,6(69,1-77,6)$ & $94,5(92,1-96,2)$ & 20,9 \\
\hline Piura & $57,2(52,8-61,6)$ & $74,3(69,9-78,3)$ & 17,1 \\
\hline Puno & $64,9(59,3-70,0)$ & $89,4(85,5-92,3)$ & 24,5 \\
\hline San Martín & $71,5(67,4-75,2)$ & $83,0(79,2-86,3)$ & 11,5 \\
\hline Tacna & $65,3(61,4-69,1)$ & $93,6(91,6-95,2)$ & 28,3 \\
\hline Tumbes & $67,2(63,7-70,5)$ & $88,2(85,7-90,3)$ & 21,0 \\
\hline Ucayali & $70,7(66,6-74,5)$ & $89,0(85,9-91,5)$ & 18,3 \\
\hline Total & $67,5(66,3-68,6)$ & $85,8(84,9-86,7)$ & 18,3 \\
\hline
\end{tabular}

* Sí: Vacunación (con fecha) consignada en la tarjeta de vacunación + vacunación (sin fecha) consignada en la tarjeta de vacunación + reporte de la madre No: No sabe + No en educar a las mujeres gestantes, así como entrenar a los promotores de salud con la finalidad de mejorar el vínculo y cercanía entre la comunidad y los establecimientos de salud ${ }^{18}$.

Nuestra investigación presentó las siguientes limitaciones: estimó la proporción de recién nacidos que recibieron la dosis contra la HvB entre 2011 al 2015, por ello, no se estimó una cobertura de vacunación por año, lo cual permitiría evaluar tendencias en el tiempo y comparar dichos resultados con otros estudios que realizan evaluaciones anuales; el estudio fue realizado en una muestra representativa de mujeres en edad fértil (MEF) que hayan tenido un nacido vivo, sin embargo dicha aproximación metodológica ha sido empleada en otros estudios $^{11,14}$; al ser un estudio de fuente secundaria, no se pudo evaluar la cobertura en algunos subgrupos de importancia, incluyendo factores susceptibles de ser modificados para mejorar dicha cobertura; por último, no se pudo evaluar la hora exacta dentro de las primeras 24 en la cual la vacunación fue realizada.

En conclusión, siete de cada diez recién nacidos de MEF peruanas recibieron la vacuna contra la HvB al nacimiento. Dicha proporción varió según región, siendo Huánuco, Apurímac, Huancave- 
lica y Ayacucho aquellas con coberturas superiores a $80 \%$. Estas estimaciones fueron realizadas considerando la información de las tarjetas de vacunación, lo cual reduce la posibilidad de sesgos de medición por autoreporte. Las coberturas estimadas a nivel nacional y regional han alcanzado la meta del 50\% para el 2020 establecida por la OMS, y nos encontramos más cerca del $90 \%$ de la meta establecida para el 2030. Es importante resaltar que encontramos una mayor cobertura en determinados subgrupos como entre aquellos que nacieron en establecimientos de salud del sector público. Recomendamos implementar estrategias para incrementar la cobertura prestacional de servicios incluyendo el parto institucional y con ello mejorar la cobertura de la inmunización al nacer.

\section{REFERENCIAS BIBLIOGRÁFICAS}

1. Resolución aprobada por la Asamblea General el 25 de septiembre de 2015. 70/1. Transformar nuestro mundo: la Agenda 2030 para el Desarrollo Sostenible. Naciones Unidas A/RES/70/1. 2015.

2. Organización Mundial de la Salud [Internet]. Estrategia mundial del sector de la salud contra las hepatitis viricas, 2016-2021. WHO. [citado el 20 de febrero de 2018]. Disponible en: http://www.who.int/ hepatitis/strategy2016-2021/ghss-hep/es/

3. World Health Assembly [Internet]. Sixty-seventh World Health Assembly: agenda item 12.3 hepatitis. Geneva: World Health Assembly, 24 May 2014. WHA67.6. [citado el 20 de febrero de 2018]. Disponible en: http://www.wpro.who.int/hepatitis/ wha67_r6-en.pdf
4. Requirements for global elimination of hepatitis B: a modelling study - The Lancet Infectious Diseases [Internet]. [citado el 19 de febrero de 2018] Disponible en: http://www.thelancet.com/journals/ laninf/article/PIIS1473-3099(16)30204-3/fulltext

5. Ramírez-Soto MC, Ortega-Cáceres G, Cabezas C. Trends in mortality burden of hepatocellular carcinoma, cirrhosis, and fulminant hepatitis before and after roll-out of the first pilot vaccination program against hepatitis B in Peru: An analysis of death certificate data. Vaccine. 2017; 35(31):3808-12 DOI: 10.1016/j.vaccine.2017.05.086. Disponible en: http://linkinghub.elsevier.com/retrieve/pii/ S0264410X17307569

6. Ministerio de Salud del Perú. NTS N ${ }^{\circ} 080$ - MINSA DGIESP V.04. Norma Técnica de Salud que establece el esquema nacional de vacunación. Resolución Ministerial N651-2016/MINSA

7. Organización Mundial de la Salud [Internet] Implementation of hepatitis B birth dose vaccination - worldwide, 2016. [citado el 20 de febrero de 2018]. Disponible en: http://apps.who.int/iris/ handle/10665/260208

8. Ropero A, Pérez-Vilar S, Pacis-Tirso C, Contreras M El Omeiri N, Ruiz-Matus C, et al. Progress in vaccination towards hepatitis $\mathrm{B}$ control and elimination in the Region of the Americas. BMC Public Health 2017: 17(1):325. DOI: 10.1186/s12889-017-4227-6

9. World Health Organization [Internet]. Global hepatitis report, 2017. WHO. [citado el 19 de febrero de 2018]. Disponible en: http://www.who.int/hepatitis/ publications/global-hepatitis-report2017/en/

10. Borda A, Florián Á, Montalván E, Dedios M, Cabezas C, Donaires F. Vacunación contra el virus de la hepatitis $\mathrm{B}$ a recien nacidos en hospitales de Lima y Callao. Resúmenes del XI Congreso Científico Internacional del INS. Lima: Instituto Nacional de Salud. 2017.

11. Cutts FT, Claquin P, Danovaro-Holliday MC, Rhoda DA. Monitoring vaccination coverage: Defining the role of surveys. Vaccine. 2016; 34(35):4103-9. DOI: 10.1016/j.vaccine.2016.06.053. Disponible en: http://linkinghub.elsevier.com/retrieve/pii/ S0264410X16304777

12. Lim SS, Stein DB, Charrow A, Murray CJL. Tracking progress towards universal childhood immunisation and the impact of global initiatives: a system- atic analysis of three-dose diphtheria, tetanus, and pertussis immunisation coverage. Lancet. 2008; 372(9655):2031-46. DOI: 10.1016/S01406736(08)61869-3

13. Miles M, Ryman TK, Dietz V, Zell E, Luman ET. Validity of vaccination cards and parental recall to estimate vaccination coverage: A systematic review of the literature. Vaccine. 2013; 31(12):1560-8. DOI: $10.1016 /$ j.vaccine.2012.10.089. Disponible en: http://linkinghub.elsevier.com/retrieve/pii/ S0264410X12015629

14. Dionne-Odom J, Westfall AO, Nzuobontane D, Vinikoor MJ, Halle-Ekane G, Welty T, et al. Predictors of Infant Hepatitis B Immunization in Cameroon: Data to Inform Implementation of a Hepatitis B Birth Dose. Pediatr Infect Dis J. 2017; 37(1):103107. DOI: 10.1097/INF.0000000000001728. Disponible en: http://Insights.ovid.com/crossref? an $=00006454-900000000-96923$

15. Choconta-Piraquive LA, De la Hoz-Restrepo F, Sarmiento-Limas CA. Compliance with birth dose of Hepatitis $B$ vaccine in high endemic and hard to reach areas in the Colombian amazon: results from a vaccination survey. BMC Health Serv Res. 2016; 16 : 293. DOI: 10.1186/s12913-016-1542-z. Disponible en: http://bmchealthservres.biomedcentral.com/ articles/10.1186/s12913-016-1542-z

16. Wiesen E, Lagani W, Sui G, Arava J, Reza S, Diorditsa S, et al. Assessment of the hepatitis B birth dose vaccination program, Papua New Guinea, 2014. Vaccine. 2016; 34(3):367-72. DOI: 10.1016/j.vaccine.2015.11.044. Disponible en: http://linkinghub. elsevier.com/retrieve/pii/S0264410X15016862

17. Allison RD, Patel MK, Tohme RA. Hepatitis B vaccine birth dose coverage correlates worldwide with rates of institutional deliveries and skilled attendance at birth. Vaccine. 2017; 35(33):4094-8. DOI: 10.1016/j.vaccine.2017.06.051. Disponible en: http://linkinghub.elsevier.com/retrieve/pii/ S0264410X17308484

18. Li X, Heffelfinger J, Wiesen E, Diorditsa S, ValiakoIleri J, Nikuata AB, et al. Improving hepatitis B birth dose coverage through village health volunteer training and pregnant women education. Vaccine. 2017; 35(34):4396-401. DOI: 10.1016/j.vaccine.2017.06.056. Disponible en: http://www.sciencedirect.com/science/article/pii/S0264410X17308538 